Original Research Paper

\title{
Tailoring the Ruggiero-Klinghardt Protocol to Immunotherapy of Autism
}

\author{
${ }^{1}$ Nicola Antonucci, ${ }^{2}$ Dietrich Klinghardt, ${ }^{3}$ Stefania Pacini and ${ }^{3}$ Marco Ruggiero \\ ${ }^{1}$ Biomedical Centre for Autism Research and Treatment, Bari, Italy \\ ${ }^{2}$ Sophia Health Institute and Klinghardt Academy, Woodinville, WA USA \\ ${ }^{3}$ Silver Spring Sagl, Arzo-Mendrisio, Switzerland
}

\author{
Article history \\ Received: 02-03-2019 \\ Revised: $17-04-2019$ \\ Accepted: 24-04-2019 \\ Corresponding Author: \\ Marco Ruggiero \\ Silver Spring Sagl, Arzo- \\ Mendrisio, Switzerland \\ Email: info@bravo-europe.com
}

\section{Introduction}

The Ruggiero-Klinghardt Protocol (RK Protocol) described in 2017 in the American Journal of Immunology (Ruggiero and Klinghard 2017), was developed with the goal of improving the sensitivity of diagnosis and efficacy of therapy in chronic conditions. Although this protocol was originally developed for persistent Lyme disease, we realized that the full protocol, or parts of it, may be useful in other chronic conditions, including autism, with particular reference to optimization of immunotherapeutic approaches. We recently described clinical cases of autism successfully treated with an immunotherapeutic approach that yielded significant results leading to complete normalization of

\begin{abstract}
Here, we describe the adaptation to the field of autism of an original procedure denominated the "Ruggiero-Klinghardt Protocol" (RK Protocol), a procedure that represents a paradigm change with significant implications for chronic conditions where immunotherapy may prove effective; that is from silent infections to neurodegenerative diseases, autism and cancer. In the context of autism, the modified RK Protocol that we propose here serves the purpose of discovering hidden infections that may be associated with autism and contribute to its symptoms. This notion is consistent with the observation that immune modulating molecules are effective in autism treatment. In the RK Protocol modified for autism, we intuce the Autism Treatment Evaluation Checklist (ATEC), a more objective and sophisticated method of evaluation compared with the Clinical Global Impression of Improvement scale that was previously used, independently of the RK Protocol, to evaluate the effectiveness of innotherapy of autism. The modifications that we present in this study take advantage of the experience that has accumulated in the two years after the publication of the original RK Protocol. Similarly to the original RK Protocol, this new version offers the advantage of being safe and relatively insensive since it does not require sophisticated instruments; because of this, it can be implemented in different parts of the world. We envisage that implementation of the RK Protocol modified for autism may contribute to and treatment on a large scale.
\end{abstract}

Keywords: Ultrasound, Autonomic Response Testing, Immune System, Imaging, Neuroinflammation, Autism some hallmark symptoms of autism (Antonucci et al., 2019a). Here we propose an adaptation of the RK Protocol to immunotherapy for autism. The protocol that will be illustrated below in detail is composed of a sequence of diagnostic and therapeutic procedures that aim at increasing sensitivity and specificity of diagnosis and at assessing and optimizing the efficacy of treatments. The diagnostic arm of the protocol described here is based on Autonomic Response Testing (ART) and diagnostic ultrasonography. ART is a manual biofeedback technique developed by Dr. Klinghardt that has the objective of evaluating the presence and/or persistence of spirochete and other infectious and noninfectious noxae that may be found in conjunction with and possibly responsible for, persistent Lyme disease. 
ART may be considered an evolution and an improvement of the approach that was proposed by Omura in 1985 and validated in several trials that include two randomized-order blinded registered clinical trials. ART developed by Dr. Klinghardt is currently utilized by scores of independent researchers for diagnosis and treatment of a variety of diseases ranging from Lyme to cancer (for details and references pertaining to ART, please see Ruggiero and Klinghardt, 2017). As far as the use of ultrasonography in the present protocol is concerned, this technique can be utilized to refine the diagnostic hypotheses suggested by ART or it can be performed independently of ART. In the latter case, here we describe the areas of the body that we recommend to study because of their potential involvement in the pathogenesis of autism. Among the organs and systems to be evaluated by ultrasonography, the brain and the meninges, examined by transcranial ultrasonography as originally reported in Bradstreet et al. (2014), are of utmost importance as evidence accumulates linking autism with impaired circulation of meningeal lymph and consequent accumulation of extraaxial fluid inside the cranial cavity (Bradstreet et al., 2014; 2015; Shen et al., 2018). It is worth noticing that transcranial ultrasonography has a number of advantages over Magnetic Resonance Imaging (MRI) that is the technique recently utilized by Shen et al. (2018) to study extra-axial fluid accumulation in children at risk for autism. At variance with MRI, transcranial ultrasonography does not require sophisticated instruments or dedicated structures, is relatively inexpensive, is simpler and faster and can be performed even on hyperactive children without the need of sedation. In addition, it provides results in real time, can be easily repeated to evaluate progression of the disease or results of treatments and, thanks to the portability of modern ultrasound systems, it can be performed in places where MRI machines are not available. In short, the transcranial ultrasonography that we first described in Ruggiero et al. (2013) and was then applied to the field of autism (Bradstreet et al., 2014), is a much more versatile technique that can be performed with ease on a high number of subjects in any part of the world.

\section{Protocol}

\section{Human Subjects}

Please notice: All methods described here, are routinely implemented as common medical procedures. No experimental procedure, either diagnostic or therapeutic is described in this protocol. The originality consists in the logic and in the sequence of the application of procedures. All procedures described in the protocol are to be performed by certified Therapists according to laws and rules regulating medical treatments in each Country. The procedures here proposed can be performed with common, commercially available, medical devices that are approved for medical use in an authorized medical structure.

\section{Structure of the Protocol}

The protocol is here described as a sequence of diagnostic and therapeutic steps. Each step includes several sub-steps. The order of the sequence of some sub-steps may be varied according to individual needs. For example, in step 3 "Diagnostic Ultrasonography", organs and regions of the body can be studied in a sequence different from the one here proposed. It is important, however, to study all the organs and the regions of the body listed in the step.

1. General and Autism-Specific Evaluation and Assessment

1.1. Perform traditional medical examination involving the collection of anamnesis, the study of previous laboratory and imaging results and objective examination

1.2. Perform a specific evaluation for autism. For example, the diagnostic criteria for autism adopted at the Biomedical Centre for Autism Research and Treatment are:

1.2.1. Marked impairment in the use of multiple nonverbal behaviors such as eye-to-eye gaze, facial expression, body postures and gestures to regulate social interaction

1.2.2. Failure to develop peer relationships appropriate to developmental level

1.2.3. Lack of spontaneous seeking to share enjoyment, interests or achievements with other people (e.g., by a lack of showing, bringing or pointing out objects of interest)

1.2.4. Lack of social or emotional reciprocity

1.2.5. Delay in, or total lack of, the development of spoken language

1.2.6. In individuals with adequate speech, marked impairment in the ability to initiate or sustain a conversation with others

1.2.7. Stereotyped and repetitive use of language or idiosyncratic language

1.2.8. Lack of varied, spontaneous, make-believe play or social imitative play appropriate to developmental level

1.2.9. Encompassing preoccupation with one or more stereotyped and restricted patterns of interest that is abnormal either in intensity or focus.

1.2.10. Apparently inflexible adherence to specific nonfunctional routines or rituals

1.2.11. Stereotyped and repetitive motor mannerisms (e.g., hand or finger flapping or twisting, or complex whole-body movements) 


\subsubsection{Persistent preoccupation with parts of objects}

As a practical example, the patients described in Antonucci et al. (2019a) met six or more criteria from this list and had been diagnosed by either a child neurologist or developmental psychologist, in addition to receiving the evaluation of the clinician at the Biomedical Centre for Autism Research and Treatment:

1.3. Autism Treatment Evaluation Checklist (ATEC). Review the ATEC that has to be compiled by parents before any type of intervention. The ATEC is a 77-item diagnostic assessment tool where a questionnaire, available in several different languages, is compiled by a parent. It has been demonstrated effective in evaluating interventional effects in autism as well as in following behavioral development over periods of time. It evaluates specific areas that are pertinent to the disorder such as speech/language and communication (section 1); sociability (section 2); sensory and cognitive awareness (section 3); physical/health behavior (section 4)

1.4. Collect biological samples for laboratory tests before performing ultrasonography, either diagnostic or therapeutic

1.4.1. Follow the instructions of the specialized laboratory as far as the modalities for collection of biological samples (urine, stools, blood, serum, breath) are concerned

2. Autonomic Response Testing (ART)

2.1. Perform ART to examine all the aspects pertaining to the autonomous response and not only muscle strength or resistance (for details see Ruggiero and Klinghardt, 2017)

2.2. Narrow the spectrum of diagnostic hypotheses and identify organs or region of the body that will be studied by diagnostic ultrasonography in step 3

2.3. Record the findings of ART that will be used for comparison in step 6

2.4. Repeat steps $2.1-2.3$ with a different couple of Therapist/Assistant; evaluate and record consistency and reproducibility

3. Diagnostic Ultrasonography

3.1. Use an ultrasound system with echo-color-Doppler application and with a linear and a convex transducer

3.2. Study the organs or the regions of the body indicated by ART

3.2.1. If ART is not implemented, study the following organs and record all images and measurements. Record as many images as possible at different levels of magnification
3.2.1.1. Temporal lobes of the brain through the temporal squama using a linear probe as described in Ruggiero et al. (2013)

3.2.1.1.1. Study the meninges, cortex, gray matter and extra-axial fluid looking for cortical dysplasia, extra-axial fluid accumulation and other brain anomalies as described in Bradstreet et al. (2014) and confirmed by Shen et al. (2018)

3.2.1.1.2. Figure 1 as an example of transcranial ultrasonography with the identification of cortical dysplasia

3.2.1.2. Thyroid

3.2.1.2.1. Look for homogeneity of echostructure, nodules, cysts and pattern of vascularization

3.2.1.2.2. Figure 2 as an example of a significant increase in blood flow that suggests chronic viral infections or autoimmune processes

3.2.1.3. Salivary glands and deep cervical nodes as described in Ruggiero and Klinghardt (2017). and in Antonucci et al. (2019b)

3.2.1.3.1. Look for homogeneity of echostructure and vascularization of the salivary glands and for enlarged nodes. Measure the nodes and document their vascularization and echostructure

3.2.1.4. Carotid artery, jugular vein and vagus nerve, bilaterally

3.2.1.4.1. Study arterial and venous blood flow and vagus nerve echostructure as described in Ruggiero and Klinghardt (2017)

3.2.1.4.2. Figure 3 as an example of peculiar blood flow in the carotid artery

3.2.1.5. Spleen and abdominal organs as described in Ruggiero and Klinghardt (2017)

3.2.1.5.1. Study dimensions, morphology and echostructure of the spleen as well as blood flow in the hilum and periphery

3.2.1.5.2. Figure 4 as an example of normal morphology and echostructure of the spleen showing normal blood flow

3.2.1.6. Look for abnormalities in morphology, dimensions, echostructure, vascularization and blood-flow of abdominal organs

4. Application of Therapeutic Ultrasound

4.1. Select the appropriate pulsed sequence, frequency and duration of treatment

4.1.1. For the spleen select pulsed sequence indicated as $50 \%$, frequency of $1 \mathrm{MHz}$, for $3 \mathrm{~min}$ 
4.1.2. For the deep cervical nodes and the vagus nerve, pulsed sequence indicated as $20 \%$, frequency of $3.3 \mathrm{MHz}$, for 90 seconds on each side of the neck

4.1.3. For other nodes identified by ART and/or ultrasonography, pulsed sequence indicated as $20 \%$, frequency of $3.3 \mathrm{MHz}$, for 90 seconds in correspondence of each node.

4.1.4. For the brain, pulsed sequence indicated as $10 \%$, frequency of $3.3 \mathrm{MHz}$, for 90 seconds on each side of the head using the temporal acoustic window as described in Ruggiero et al. (2013) and Bradstreet et al. (2014)
4.2. Apply therapeutic ultrasound with slow circular movements in order to direct the ultrasound waves to the targeted organ or structure. Use abundant gel

4.3. Invite the patient to exercise or to breathe slowly and deeply for about 5 minutes after the last application of therapeutic ultrasound.

5. Manual Lymphatic Drainage.

5.1. Perform manual lymphatic drainage as described in Antonucci et al. (2019b).

5.2. Instruct parents or tutors to perform manual lymphatic drainage every night before sleep.

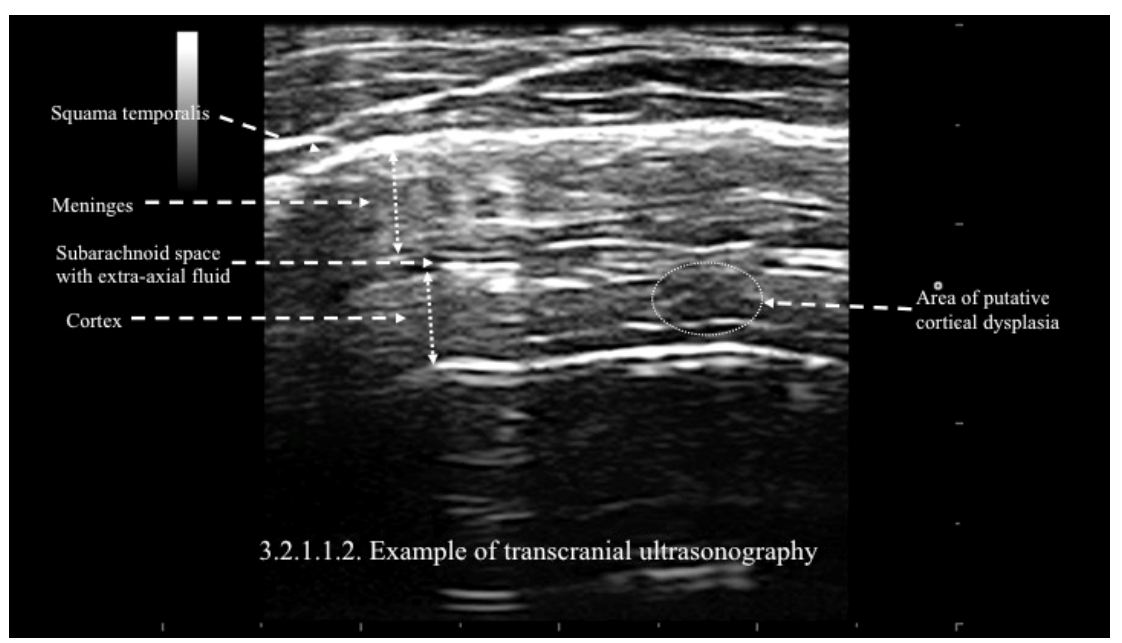

Fig. 1: Transcranial ultrasonography. Squama temporalis, meninges, subarachnoid space with extra-axial fluid and cortex are visible. On the right, a putative lesion compatible with cortical dysplasia as described in Bradstreet et al. (2014) can be appreciated. This figure refers to step 3.2.1.1.2. of the protocol. This image is from the personal archive of one of the Authors and is presented as an example of transcranial ultrasonography; it does not refer to any of the clinical cases mentioned in this study

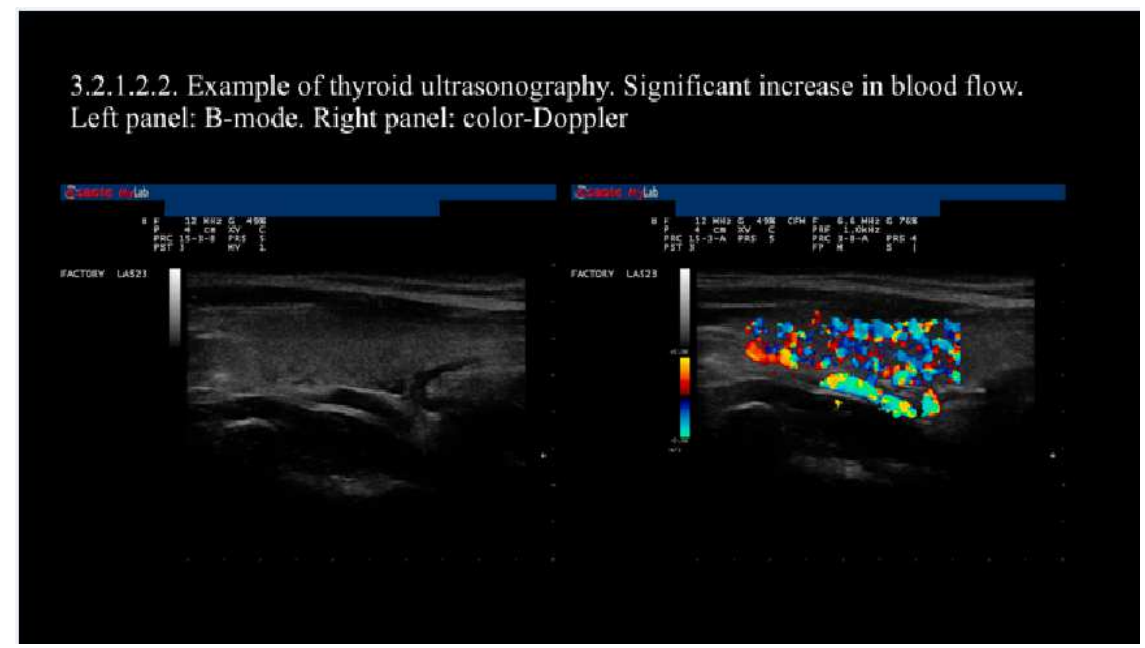

Fig. 2: Ultrasonography of the thyroid. In the left panel, the longitudinal projection of the thyroid lobe in B-mode shows an inhomogenous, rather hypoechoic appearance. In the right panel, echo-color-Doppler shows abnormal increase in blood flow. This finding is compatible with inflammatory processes that, in the case of the thyroid, are often due to viral or autoimmune noxae. This figure refers to step 3.2.1.2.2. of the protocol. This image is from the personal archive of one of the Authors and is presented as an example of ultrasonography of the thyroid; it does not refer to the any of the clinical cases mentioned in this study 


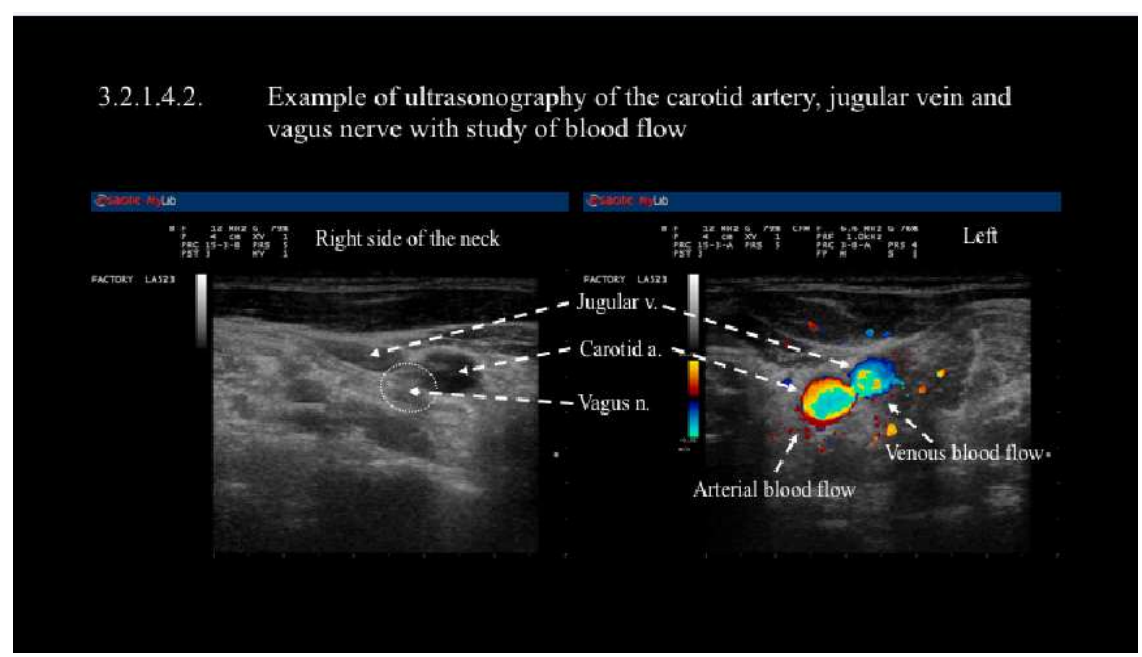

Fig. 3: Ultrasonography of the neck: carotid artery, jugular vein, vagus nerve. Left panel: Right side of the neck, B-mode. The carotid artery and the jugular vein are clearly visible. The vagus nerve appears as a small triangular structure located posteriorly inside the carotid sheath between the common carotid artery and the internal jugular vein. Right panel: Left side of the neck, echo-color-Doppler. Blood flow in the carotid artery and jugular vein is clearly visible. Arterial blood flow appears red whereas venous blood flow appears blue according to the rule of thumb designated Blue Away Red Towards (BART). The blue area inside the carotid artery may indicate a condition known as pulsus bisferiens. This figure refers to step 3.2.1.4.2. of the protocol. This image is from the personal archive of one of the Authors and is presented as an example of ultrasonography of the neck with study of blood flow; it does not refer to the any of the clinical cases mentioned in this study

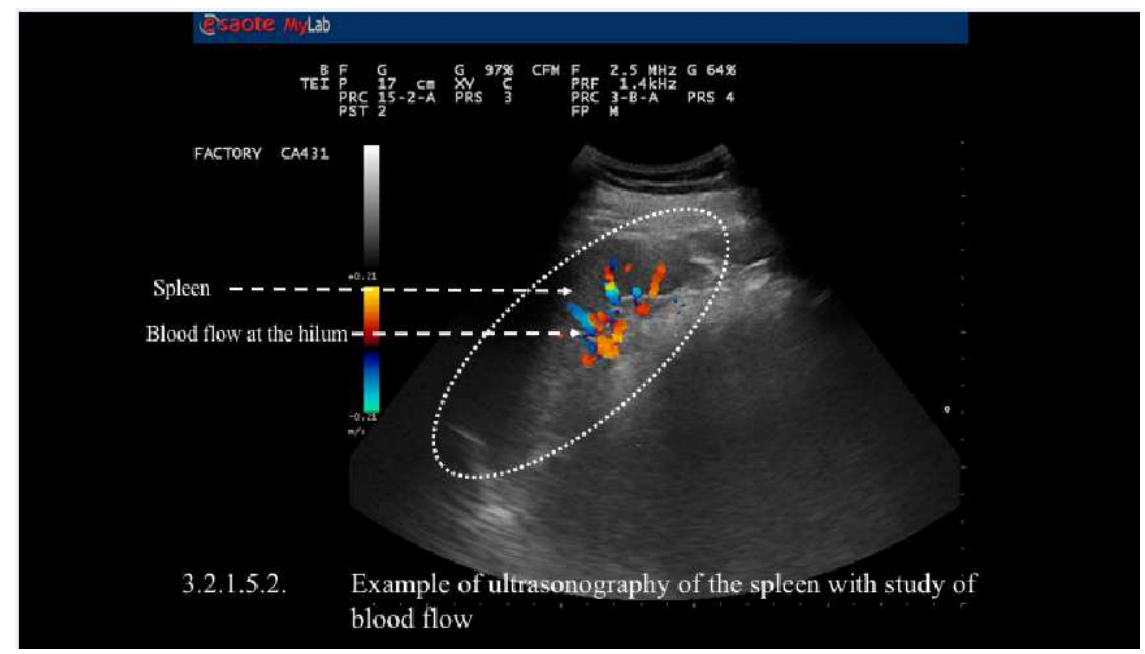

Fig. 4: Ultrasonography of the spleen. In this example, dimensions, morphology, echostructure and pattern of vascularization of the spleen appear normal. This figure refers to step 3.2.1.5.2. of the protocol. This image is from the personal archive of one of the Authors and is presented as an example of ultrasonography of the spleen with study of blood flow; it does not refer to the any of the clinical cases mentioned in this study

\section{Second ART.}

6.1. Perform ART; compare the results with those obtained in step 2 and record the results and the comparisons.

6.2. Evaluate and record consistency and reproducibility with a different couple of Therapist/Assistant and compare the results with those obtained in step 2.4

\section{Biological Sample Collection.}

7.1. Six hours after step 4, collect biological samples as in step 1.4

\section{Individualized Treatment}

8.1. Treat the patient according to the standard of care indicated for each specific condition identified with the previous steps 
8.2. Perform ART to fine-tune the choice of specific drugs, supplements or procedures

8.2.1. Confirm the potential efficacy of the treatment in the preceding step, using diagnostic ultrasonography as described in the following step

8.2.1.1. Find below examples of effects to be expected using an immunotherapeutic approach (imuno ${ }^{\circledR}$ ) as described in Antonucci et al. (2019a)

8.2.1.1.1. Increase in splenic blood flow due to activation of macrophages with the release of nitric oxide as described in Ruggiero et al. (2014)

8.2.1.1.2. Reduction of signs of neuroinflammation as observed by transcranial ultrasonography (step 3.2.1.1.1.).

8.3. Apply targeted therapeutic ultrasound as described in step 4 with the goal of exploiting the known therapeutic effects of pulsed ultrasound that comprise anti-inflammatory effects, enhanced lymphatic drainage and optimization of drug uptake and utilization.

9. Evaluation of Efficacy and Assessment of End-point

9.1. Repeat steps 1 to 8 to evaluate the efficacy of the treatment and its end-point

9.1.1. Second ATEC. Review ATEC compiled after implementation of this protocol or other therapeutic intervention. In the following substeps, examples of results evaluated by ATEC using an immunotherapeutic approach (imuno ${ }^{\circledR}$ ) as described in Antonucci et al. (2019a) are reported. In this example, the second ATEC was performed after 8 weeks of treatment with imuno ${ }^{\circledR}$

9.1.1.1. "... the behavioral symptoms described by the queries "bed-wetting", "wets pants/diapers", "soils pants/diapers", "diarrhea", "constipation", "eats too much/too little", "not sensitive to pain" improved from "moderate" to "not a problem", thus indicating complete normalization of these very significant symptoms of autism." (Antonucci et al., 2019a).

9.1.1.2. "... the behavioral symptoms described by the queries "shows no affection", "fails to greet parents", "avoids contact with others", "dislikes being held/cuddled" improved from "very descriptive" to "not descriptive", thus indicating complete normalization of these significant symptoms of autism." (Antonucci et al., 2019a).

9.1.1.3. "... In section 2 , that is the subscale for sociability, improvements were observed for the symptoms described by the queries "does not imitate", "disagreeable/not compliant", "indifferent to being liked" where the answers showed improvement from "somewhat descriptive" to "not descriptive", thus indicating normalization of behaviors." (Antonucci et al., 2019a)

9.1.2. Adapt and/or modify the therapeutic approach based on review of ATEC and other results

\section{Discussion}

Three of the critical steps in the original RK Protocol as described in Ruggiero and Klinghardt (2017) were represented by ART, diagnostic and therapeutic ultrasound. ART was used to achieve different purposes. Thus, the initial ART had the goal to identify the organs or the regions of the body that required further investigation; to narrow the diagnostic hypotheses and to provide information on the underlying pathology i.e., the presence of pathogens, neoplastic cells, abnormal cells or toxicants. ART was then repeated; this second ART had the scope of evaluating whether therapeutic ultrasound had been successful in mobilizing pathogenic noxae from sanctuaries or reservoirs making them "visible" to the Therapist performing ART. Thirdly, ART for specific patient treatment served the purpose of finetuning the therapy. Another critical step was represented by the use of therapeutic ultrasound that, in the context of the original RK Protocol, had the role to "squeeze" at the cellular and molecular level the organs or the tissues that may have offered a place to hide to pathogens or other noxae. The original RK Protocol was designed as a recursion of diagnostic procedures that served to confirm with each other and the goal of achieving accurate and early diagnosis in elusive conditions and provide for individualized treatment.

The original RK Protocol borrowed the "shock and kill" approach that is used to eliminate the reservoirs of HIV that are responsible for the latency and persistence of the virus. The "shock and kill" strategy pursues the goal of stimulating HIV replication in a latent viral reservoir; at first sight, such a strategy may appear counterintuitive as the objective of pharmacological antiretroviral therapies is to block, not to stimulate, HIV replication. However, the rationale behind this approach, as in the RK Protocol, is to render the hidden virus "visible" to the immune system and to the chemical drugs (Melkova et al., 2017). Thus, the scope of the original RK Protocol was to render pathogens, toxicants, neoplastic cells or cells infected by viruses that would otherwise be inaccessible to diagnostic and therapeutic tools, "visible" so that they can be identified and fought by the Therapist and by the body's immune system.

When the original RK Protocol was developed, it was envisaged that future applications would have been in the field of neurodegenerative and neurodevelopmental conditions with particular reference to autism. In the context of autism, the modified RK Protocol that we 
propose here might serve the purpose of discovering hidden infections that may be associated with autism and contribute to its symptoms. This notion, in conjunction with the observation that immune modulating molecules were effective in autism, can be found in the seminal paper by Bradstreet et al. (2012). In 2019, we were able to confirm those results with a more potent compound, (imuno $^{\circledR}$ ) that yielded impressive clinical results. At variance with the paper by Bradstreet et al. (2012) our results in 2019 were confirmed by the ATEC, a more objective and sophisticated method of evaluation compared with the Clinical Global Impression of Improvement scale that was used in 2012. It was based on this observation that we decided to include the ATEC in the modified RK Protocol adapted for autism that is here described. In addition, it is worth noticing that the effects of immune modulating strategies that work on the immune-neuro-endocrine axis, such as imuno ${ }^{\circledR}$, may be slow and progressive; since these approaches function by rebalancing physiological mechanisms, the effects may go unnoticed unless specifically addressed. The ATEC has been introduced in the modified RK Protocol precisely to address these aspects since it provides a useful tool to objectively assess the efficacy of the treatment.

The original RK Protocol published in 2017 represented a novelty in the field of diagnostics and therapeutics because it aimed at achieving a higher degree of precision by combining in an integrated and logically sequential manner, techniques and procedures that have been used for decades. The modifications that we present in this study are meant to tailor the protocol to the specific field of autism and take advantage of the experience that has accumulated in the two years after the publication of the original RK Protocol. Similarly to the original RK Protocol, this new version offers the advantage of being safe and relatively inexpensive since it does not require sophisticated instruments or dedicated structures; because of this, it can be easily implemented in any part of the world.

The latter consideration bears relevance in the context of prevention, early diagnosis and treatment of autism. As demonstrated by Shen et al. (2018), accumulation of cerebrospinal extra-axial fluid is a reliable brain anomaly that can be observed relatively early, that is before the onset of clinical symptoms. Study of extra-axial fluid accumulation may serve the purpose of identifying children at risk for developing autism, thus enabling implementation of early interventions aimed at preventing the development of the disease. Among suitable interventions, the immunotherapeutic approaches described by Antonucci et al. (2019a; 2019b) appear most promising as they address the major pathogenetic factors responsible for the symptoms of autism. However, the majority of clinical settings and therapists have no easy access to MRI for studying extra-axial fluid accumulation, thus limiting applicability of this technique to large-scale prevention, early diagnosis and treatment of autism. The RK Protocol modified for autism, on the contrary, can be implemented in every medical office of the world as it does not require expensive or sophisticated instruments but a common ultrasound system that has the additional advantage of being portable. We envisage that implementation of the RK Protocol modified for autism may contribute to decrease the burden of the disease as it enables prevention, early diagnosis and treatment on a large scale.

\section{Acknowledgement}

The Authors wish to thank the parents of the autistic children whose cases are reported here for their priceless collaboration. The Authors wish to thank Ms. Daniela Deiosso for inspiring discussion and relentless support and the Therapists at the Sophia Health Institute for their precious collaboration. The Authors wish to express their gratitude for the human and scientific legacy of Dr. Bradstreet whose insight inspires their work.

\section{Conflict of Interest}

Nicola Antonucci is the founder of the Biomedical Centre for Autism Research and Treatment, a private clinic. Dietrich Klinghardt is the inventor of ART and the founder of the Klinghardt Institute, the Klinghardt Academy, the Institut fuer Neurobiologie and the Sophia Health Institute, a private clinic. Dr. Klinghardt consults for several companies producing supplements and other remedies. Marco Ruggiero is the inventor of a number of supplements and, together with Stefania Pacini, developed the supplement mentioned in this study (imuno ${ }^{\circledR}$ ). Neither he, nor Dr. Pacini, had any prior knowledge of the therapies being used, nor of the details of any patient whose clinical outcomes are summarized in this study. Marco Ruggiero is member of the Editorial Board of The American Journal of Immunology and is waived from the Article Processing fee for this contribution; he receives no remuneration for his editorial work.

\section{Authors' Contribution}

Nicola Antonucci: Performed all the diagnostic and therapeutic procedures whose results are described in this study.

Dietrich Klinghardt: Is the inventor and developer of ART.

Marco Ruggiero and Stefania Pacini: Wrote the first draft of this paper, provided critical input and assisted in revising and improving the paper. 


\section{Advisory}

No information in this paper is presented by the Authors as direct medical advice to any individual. Caregivers, researchers and interested parties should research all information given. Beginning any significant biomedical or other interventions that may impact physiology or making changes to an established regimen should be discussed with the patient's physician in advance. Standard of care for each pathology must be followed as well as rules and regulations established by Health Authorities of each Country.

\section{References}

Antonucci, N., S. Pacini and M. Ruggiero, 2019a. Clinical experience of integrative autism treatment with a novel type of immunotherapy. Madridge J. Vaccines, 3: 71-76.

DOI: $10.18689 / \mathrm{mjv}-1000116$

Antonucci, N., S. Pacini and M. Ruggiero, 2019b. Clinical experience of integrative autism treatment with manual lymphatic drainage. EC Neurol., 11: 21-28.

Bradstreet J.J., E. Vogelaar and L. Thyer, 2012. Initial observations of elevated alpha-nacetylgalactosaminidase activity associated with autism and observed reductions from GC proteinmacrophage activating factor injections. Autism Insights, 4: 31-38. DOI: 10.4137/AUI.S10485

Bradstreet, J.J., M. Ruggiero and S. Pacini, 2015. Commentary: Structural and functional features of central nervous system lymphatic vessels. Front. Neurosci., 9: 485-485.

DOI: $10.3389 /$ fnins.2015.00485
Bradstreet, J.J., S. Pacini and M. Ruggiero, 2014. A new methodology of viewing extra-axial fluid and cortical abnormalities in children with autism via transcranial ultrasonography. Front. Hum. Neurosci., 15: 7:934. DOI: 10.3389/fnhum.2013.00934. PMID: 24459462

Melkova, Z., P. Shankaran, M. Madlenakova and J. Bodor, 2017. Current views on HIV-1 latency, persistence and cure. Folia Microbiol. (Praha), 62: 73-87. DOI: 10.1007/s12223-016-0474-7, PMID: 27709447

Ruggiero, M. and D. Klinghard, 2017. The RuggieroKlinghardt (RK) protocol for the diagnosis and treatment of chronic conditions with particular focus on Lyme disease. Am. J. Immunol., 13: 114-126. DOI: 10.3844 /ajisp.2017.114.126

Ruggiero, M., E. Ward, R. Smith, J.J. Branca and D. Noakes et al., 2014. Oleic acid, deglycosylated vitamin D-binding protein, nitric oxide: A molecular triad made lethal to cancer. Anticancer Res., 34: 3569-3578. PMID: 24982371

Ruggiero, M., S. Magherini, M.G. Fiore, B. Chiarelli and G. Morucci et al., 2013. Transcranial sonography: A technique for the study of the temporal lobes of the human and non-human primate brain. Ital. J. Anat. Embryol., 118: 241-55. PMID: 24640587

Shen, M.D., C.W. Nordahl, D.D. Li, A. Lee and K. Angkustsiri et al., 2018. Extra-axial cerebrospinal fluid in high-risk and normal-risk children with autism aged 2-4 years: A case-control study. Lancet Psychiatry, 5: 895-904.

DOI: $10.1016 / \mathrm{S} 2215-0366(18) 30294-3$ 